$$
\begin{array}{r}
\text { POPULAÇ } \\
\text { TRADICION } \\
\text { UMA REFLE } \\
\text { SOBRE A QUES } \\
\text { NUTRICIO }
\end{array}
$$




\section{POPULAÇÕES TRADICIONAIS: UMA REFLEXÃO SOBRE A QUESTÃO NUTRICIONAL}

UNIVERSIDADE FEDERAL DO PARÁ 


\title{
POPULAÇÕES TRADICIONAIS: UMA REFLEXÃO SOBRE A
} QUESTÃO NUTRICIONAL

\section{Resumo}

Este artigo tem por objetivo fomentar reflexões sobre a questão nutricional diante das especificidades das populações tradicionais da Amazônia brasileira. Para tal, discorremos sobre temáticas que norteiam o campo da bioética quanto à vulnerabilidade, ao processo de transição nutricional, ao direito humano à alimentação adequada, além das experiências de vivência acadêmica que nos conduziram a reflexões do tema.

Palavras-chave: Bioética, nutrição, comportamento alimentar.

TRADITIONAL POPULATIONS: A REFLECTION ON THE NUTRITIONAL ISSUE

\begin{abstract}
This paper aims to foster reflections on the nutritional issue in view of the specificities of the traditional populations of the Brazilian Amazon. In order to do this, we discuss topics that guide the field of bioethics as regards vulnerability, the process of nutritional transition, the human right to adequate food, and the experiences of academic livingness that led us to reflect on the theme. Keywords: Bioethics, nutrition, food behavior.
\end{abstract}




\section{POBLACIONES TRADICIONALES: UNA REFLEXIÓN SOBRE LA CUESTIÓN NUTRICIONAL}

\section{Resumen}

Este artículo tiene por objetivo fomentar reflexiones sobre la cuestión nutricional ante las especificidades de las poblaciones tradicionales de la Amazonia brasileña. Para ello, discoutimos sobre temáticas que orientan el campo de la bioética en cuanto a la vulnerabilidad, al proceso de transición nutricional, al derecho humano a la alimentación adecuada, además de las experiencias de vivencia académica que nos condujeron a reflexiones del tema. Palabras clave: Bioética, nutrición, comportamiento alimentario

Simone Dora Silva da Silva simone.dora@hotmail.com

Regiane Padilha dos Santos regianepadilha0@gmail.com 


\section{INTRODUÇÃO}

São inúmeros os desafios da atuação profissional após a formação acadêmica, quando se fala em grupos específicos, seja recém-nascidos, grávidas, esportistas ou idosos, mas ao se debater sobre povos e comunidades tradicionais, o conhecimento ínfimo a respeito de suas práticas culturais, hábitos alimentares e organização social torna o desafio ainda maior de ser transposto para a resolução das demandas advindas desta população.

Conhecer as especificidades, a história destes povos e suas crenças é de fundamental importância para que as intervenções a serem realizadas não representem impactos nos seus hábitos e costumes. Entender que, além de peculiaridades, existe também uma identidade cultural a ser respeitada é a chave para o sucesso de nossa laboração (Conti \& Souza 2013:780-804).

É significativo ainda considerar que a atuação do profissional de saúde precisa garantir o acesso a direitos humanos fundamentais, como por exemplo saúde, educação, assistência, alimentação adequada e de qualidade, ofertando a proteção devida àquele que precisa, garantindo que sua insegurança não seja alvo de violações (BRASIL 2010).

Posto isto, este trabalho visa uma reflexão sobre à luz dos pressupostos bioéticos que permeiam o campo nutricional em populações tradicionais na Amazônia.

\section{POVOS E COMUNIDADES TRADI- CIONAIS NO BRASIL E QUESTÕES BIOÉTICAS}

O decreto $n^{\circ}$ 6040/2007 conceitua Povos e Comunidades Tradicionais (PCT) como:

grupos culturalmente diferenciados e que se reconhecem como tais, que possuem formas próprias de organização social, que ocupam e usam territórios e recursos naturais como condição para sua reprodução cultural, social, religiosa, ancestral e econômica, utilizando conhecimentos, inovações e práticas gerados e transmitidos pela tradição (Brasil 2007).

Tal descrição abrange diversos grupos étnicos, dentre eles os mais conhecidos são os indígenas, os quilombolas e comunidades como os ribeirinhos, pescadores, e demais, que, por possuírem características peculiares, são englobados na nomenclatura de populações tradicionais (P'T) (BRASIL 2010; Rebelo 2014:75-84).

É importante destacar que a nível internacional o termo PT é remetido a populações indígenas, mas nacionalmente, por possuir uma gama de culturas e modos de vida que se enquadram no termo tradicional, a denominação PT ganha uma nova dimensão abarcando mais povos além dos índios, mas também os seringueiros, os extrativistas, os povos da floresta, os faxinais, e outros. Percebe-se que os indígenas possuem peculiaridades que os distingue dos restantes, como por exemplo serem tutelados a uma Fundação $\mathrm{Na}$ cional, subordinada ao Governo Federal, e suas gerações passadas já estarem 
presentes em terras brasileiras desde a era pré-colombiana (Rebelo 2014: 7584; Conti \& Souza 2013:780-804).

Historicamente, percebe-se uma diferenciação de comunidades indígenas e quilombolas de outros PCT, uma vez que politicamente houve reconhecimento destas já na Constituição de 1988. Os demais povos somente conseguiram reconhecimento após este período. Observa-se ainda que a trajetória de afirmação da população indígena ainda é anterior a 1988, quando já em 1967 se cria a Fundação Nacional do Índio (FUNAI), que visa proteger e ofertar auxílio nos diversos campos seja na saúde, educação ou assistência (Conti \& Souza 2013:780-804; Garnelo \& Pontes 2012).

Há que ter em conta, ainda, que o caminho para chegar à definição de PCT foi longo e passou por várias mudanças, uma vez que entender e enquadrar inúmeros povos em uma classificação não pode ser considerado tarefa simples, os contextos e características que permeiam cada grupo não possuem um único direcionamento, tampouco um uno objetivo. No decorrer das evoluções políticas ocorridas em território brasileiro entre os anos de 1990 a 2007, houve um primeiro Decreto, posterior Portaria, subsequente Medida Provisória, sequente Lei até se chegar ao Decreto mais atual de $n^{\circ} 6040$ de fevereiro de 2007, que se propõe a delinear o que vêm a ser povos e comunidades tradicionais, mas não se pode entender este debate como encerrado, à medida que os traços culturais destas populações são continuamente reafirmados e reelaborados. Vários autores discor- rem sobre o tema, mas percebe-se ao estudá-los o quanto é difícil dar um fechamento a esta questão, seja pela pluralidade de atributos de cada grupo, seja por sua diversidade de costumes que não podem e nem devem ser agregados (Conti \& Souza 2013:780-804).

Ainda assim, levando em conta os discursos que abordam o assunto, é possível criar um norte que permita caracterizar minimamente estes grupos. Observa-se, por exemplo, que tais comunidades compartilham seu território tendo a posse, seja definitiva ou transitória e, fazendo uso coletivo da mesma, utilizam-se de técnicas ambientais de baixo impacto, têm seus papéis sociais estabelecidos de forma igualitária, algumas comunidades possuem uma liderança local que fala por todos e uma cultura que os diferencia e os identifica (Conti \& Souza 2013:780-804).

Aqui se faz necessário abrir uma lacuna, a fim de detalhar mesmo que minimamente os povos indígenas, quanto a questão do contato com não-índio, havendo uma divisão distinta, entre povos isolados, o qual a FUNAI descreve como: " a grupos indígenas com ausência de relações permanentes com as sociedades nacionais ou com pouca frequência de interação, seja com não-indios, seja com outros povos indígenas"; há também os povos indígenas de recente contato, o qual a FUNAI descreve como: "povos ou grupos indígenas que mantêm relações de contato permanente e/ ou intermitente com segmentos da sociedade nacional (...) grupos que mantêm fortalecidas suas formas de organização social e suas dinâmicas coletivas próprias, e que definem sua relação com o Estado e a sociedade nacional com alto grau de autono- 
mia”; e povos indígenas que estabeleceram contato e relação permanente com os não-índios - o qual será o objeto deste excerto - sendo possível verificar que estes já absorveram traços da influência ocidental em seus costumes e hábitos notadamente os de cunho alimentício, principalmente porque por vezes as tribos que já possuem convívio com os não-índios, estabelecem assentamento acerca de Postos Indígenas ou de Distritos Sanitários Especiais Indígenas (DSEIs) como por exemplo os Pólos Base II, o que interfere diretamente na apreensão das influências seja na nova organização de vida, seja no modo de vestir, ou de se alimentar (Amorim 2017).

Partindo de tais detalhamentos, o campo da bioética e da ética em pesquisa também entende o quão importante é considerar essas pequenas nações, ao se elaborar instrumentos que as protejam quando se discursa sobre pesquisa envolvendo seres humanos, prova disto é que a Declaração Universal sobre Bioética e Direitos Humanos (DUBDH), em seu Art. 12, assegura o Respeito pela diversidade cultural e o pluralismo. Seguindo o mesmo princípio, a Resolução n 466 de 2012, ao proferir sobre os Aspectos Éticos da Pesquisa com seres humanos, em sua alínea $\mathrm{k}$ aborda sobre o respeito aos valores culturais, sociais, e demais valores, e na alinea 1 fala sobre a garantia de que os estudos realizados em comunidades terão como meta, sempre que possível, o retorno dos benefícios para estas. Concordando com estas legislações, a Resolução n 510 de 2016 também reforça, em seu Art. $3^{\circ}$ Inciso
III, a importância do respeito quanto aos diversos valores dos participantes de pesquisa incluindo os valores culturais e ainda descreve no seu Art. 13 que, nos povos em que existe a figura de um líder, deve ser respeitado pelo pesquisador tal peculiaridade, mas tal especificidade jamais ultrapassará o consentimento do indivíduo no âmbito da pesquisa (UNESCO 2005; BRASIL 2012, 2016).

Por fim, é necessário que se tenha em nota, ao estudar grupos tradicionais, que existem atributos que não podem nem devem ser desmerecidos, e que há leis que os protegem e resguardam, sendo fundamental se pensar em investigações que tragam benefícios efetivos a estes indivíduos e grupos sociais e que representem avanços e conquistas de autonomia para a esta coletividade, sem ferir ou denegrir tradições seculares, nem de alguma forma afetar sua identidade cultural.

\section{ENTENDENDO A VULNERABILI- DADE}

De acordo com a Resolução $\mathrm{n}^{\circ}$ 466/2012, vulnerabilidade é definida como o "estado de pessoas ou grupos que, por quaisquer razões ou motivos, tenham a sua capacidade de autodeterminação reduzida ou impedida, ou de qualquer forma estejam impedidos de opor resistência, sobretudo no que se refere ao consentimento livre e esclarecido" (BRASIL 2012).

É relevante ainda compreender que a vulnerabilidade, nas pesquisas envolvendo seres humanos, pode ser uma condição temporal ou permanente, na 
medida em que se analisa as circunstâncias inerentes à pessoa ou a sua realidade social, biológica e política (Guimarães \& Novaes 1999).

Rogers e Ballantyne (2008) pontuam que a vulnerabilidade pode ser subdividida em duas classificações, a saber: 1) vulnerabilidade extrínseca, que decorre de situações externas e independentes do sujeito, ou seja, são condições em que o indivíduo está inserido, que podem ou não ser alteradas no decorrer de sua vida, como por exemplo sua condição financeira, falta de acesso a serviços de saúde, educação, dentre outros; 2) vulnerabilidade intrínseca, que se refere a características próprias da pessoa, que geralmente nasce com tais determinantes, mas também pode adquiri-los ao longo de sua existência, como exemplo temos as doenças neurológicas, crianças em condições de prematuridade, idosos debilitados e ainda doenças adquiridas no percurso da vida que incapacita o ser humano, dificultando a habilidade de decisão sobre si.

Ressalta-se, ainda, que a vulnerabilidade pode se referir a grupos de pessoas que possuam condições intrínsecas semelhantes ou ainda compartilhem condições extrínsecas que as coloquem em condições de insegurança, como exemplo temos as comunidades tradicionais, em especial os indígenas de convívio permanente que em sua hierarquia, algumas vezes respondem a uma liderança e tais comunidades na realidade brasileira, estão sob a proteção da FUNAI não sendo autônomas para definir e determinar diversas ações que correspondem ao seu cotidiano, e ain- da, a participação em pesquisas mesmo que estas tragam algum benefício a sua comunidade (Almeida 2010:537-48).

No que tange a pesquisa envolvendo seres humanos, é importante realçar que ao se estudar as populações tradicionais, com o intuito de garantir a sua integridade, é necessário se ater não somente à Resolução n 466/2012, mas também a Declaração Universal sobre Bioética e Direitos Humanos (DUB$\mathrm{DH})$, que em seu artigo $8^{\circ}$ destaca que “(...) os individuos e grupos particularmente vulneráveis devem ser protegidos (...)”. Essa declaração descreve ainda sobre a responsabilidade social e saúde em seu artigo 14, que não aparece na Resolução n 466/2012. Já na Resolução nº 510/2016, há que se atentar que tal legislação se propõe a assegurar a participação em pesquisas com o mínimo de risco para os seus participantes e substitui, ainda, uma noção reificada de vulnerabilidade pela ideia de "situação de vulnerabilidade", ampliando o entendimento dessa concepção. (Guimarães \& Novaes 1999; Almeida 2010:537-48).

Partindo destes pontos e visando resguardar os participantes de pesquisa, é necessário observar no momento da assinatura do Termo de Consentimento Livre e Esclarecido (TCLE) a utilização de linguagem acessível que explicite os objetivos da pesquisa, os resultados esperados, possíveis riscos e potenciais benefícios oriundos deste estudo para o participante e também para a sua comunidade e, ainda, descrever qual a importância da participação deste sujeito na investigação, sempre levando em conta a Declaração Universal dos Direitos Humanos (DUDH), 
no que tange o respeito à dignidade da pessoa humana (ONU 1948).

Outro fator desafiador quando discutimos vulnerabilidade é a existência do Imperialismo Moral que aparece como um impulsionador do aumento da fragilidade dos indivíduos ou populações em estudo à medida que impõe por diferentes meios os seus padrões morais a outras culturas, regiões e países. Neste sentido e desejando combater tal imperialismo, a Bioética de Intervenção (BI) tem orientado medidas com o objetivo de enfrentar esta questão, intentando proteger principalmente sujeitos e populações vulneráveis e desemponderadas, para a diminuição dos contrastes sociais, à medida que prioriza a aplicação dos quatro P (Prudência; Prevenção; Precaução e Proteção), entendendo que o reconhecimento de sua própria fragilidade é o ponto de partida para a superação e avanços (Morais \& Monteiro 2017).

Outra questão relevante a ser discutida é a utilização de estudos double standard, também conhecidos como estudos de duplo padrão ético, de pesquisas em países desenvolvidos e em desenvolvimento, a exemplo de estudos multicêntricos do ramo farmacêutico que muitas vezes se utilizam do placebo em grupos controle destes países subdesenvolvidos, para checar a eficácia de um medicamento, sendo que em sua maioria os indivíduos e grupos mais frágeis é que acabam por ser alvo destas pesquisas, sob a falsa crença de que terão acesso a tratamentos e medicamentos que normalmente não estão disponíveis na sua dada realidade.
Cabe ao pesquisador levar em consideração as características e particularidades dos grupos a serem estudados, a fim de garantir a proteção de grupos desamparados e ainda a integridade dos dados a serem coletados, realizando o mínimo de interferências e aplicando os princípios bioéticos de autonomia, beneficência, não-maleficência e justiça, garantindo assim menos riscos aos pesquisados e o máximo de resultados positivos advindos do estudo realizado para estas coletividades populacionais (COSAC 2017:19-29; Morais \& Monteiro 2017).

\section{TRANSIÇÃO EPIDEMIOLÓGICA NU- TRICIONAL}

Paralelo à condição de vulnerabilidade a que os povos tradicionais estão submetidos, as modificações epidemiológicas, demográficas e nutricionais ao longo dos anos, além de proporcionarem a diminuição dos níveis de morbidade, fecundidade, aumento da expectativa de vida, promoveram alterações nos hábitos alimentares, condição que afeta também os povos tradicionais da floresta (Soares \& Barreto 2014:341354). A transição nutricional caracteriza-se por mudanças nos problemas nutricionais de um nicho populacional, geralmente alteração de desnutrição e obesidade. Acerca da obesidade, esta se encontra em inúmeros grupos populacionais constituindo-se uma condição de risco para as doenças crônicas não transmissíveis (Mattos \& Neves 2009). Ainda no que diz respeito às mudanças decorrentes subsequentes da interação populacional, Garnelo \& Pontes 
(2012) relatam que este convívio modificou estratégias de subsistência, ocupação de territórios, recursos naturais e impactos na organização da população indígena de contato permanente e aqueles de contato recente.

\section{BIOÉTICA E DIREITO HUMANO À ALIMENTAÇÃO ADEQUADA}

A Declaração Universal dos Direitos Humanos (DUDH) diz respeito ao direito ao acesso de alimentos saudáveis suficientes e adequados de acordo com as tradições culturais de seu povo garantindo dignidade plena das dimensões física, mental, individual e coletiva. Para assegurar o objetivo da DUDH foi criada a Lei $n^{\circ} 11.346$ de 2006, que redige sobre os princípios da universalidade e equidade no acesso à alimentação adequada sem discriminação respeitando a dignidade de cada indivíduo.

O reconhecimento da dignidade humana no sentido de direito igualitário descrito na DUDH apresenta-se como marco axiológico da bioética, correspondendo como ponto de aproximação entre ela e o direitos humanos uma vez que esse princípio é fundamental na construção teórica tanto no campo da bioética quanto no campo dos direitos humanos. (Corradi-Perini et al. 2012; Declaração Universal dos Direitos Humanos 1948). No que diz respeito à alimentação adequada, o direito humano à alimentação adequada (DHAA) apresenta-se na Declaração Universal sobre Bioética e Direitos Humanos, relatado no artigo 14, que determina que os progressos científi- cos e tecnológicos devem garanti-lo.

Assim, mediante as questões referentes à garantia dos direitos da segurança alimentar dos povos tradicionais, o nutricionista é o profissional com atribuições acadêmicas específicas para observar, diagnosticar e, diante do contexto em que este indivíduo está inserido, realizar a conduta nutricional de acordo com a realidade local e os valores socioculturais do paciente, habilidades estas que o tornam valoroso para o modelo do Sistema Único de Saúde (SUS) (Matos \& Neves 2017).

Para as adversidades encontradas pelo profissional nutricionista em atuação, o diálogo com representantes do nicho populacional em que este atua apresenta-se como uma boa alternativa para a construção de parceria em busca ativa de alternativas para as resoluções de problemas que afligem essas populações (Garnelo \& Pontes 2012).

\section{PERSPECTIVA NUTRICIONAL EM COMUNIDADES E POVOS TRADI- CIONAIS DA FLORESTA}

A Amazônia é um ecossistema vasto e complexo, abrigando uma população majoritariamente urbana. Sua população rural inclui grupos tradicionais, como os indígenas e quilombolas. Suas especificidades, seu modo de vida, ainda são assuntos com lacunas a serem preenchidas e suas relações com o meio ambiente e saúde possuem perspectivas complexas e desafiantes (Silva 2006:77-94). A dimensão amazônica impressiona por suas proporções continentais, particularidade esta que 
torna seu acesso restrito e com dificuldade de estadia e locomoção. Mesmo diante de tal magnitude, em grande oportunidade uma das autoras deste artigo pôde estar inserida em estudos com as populações da floresta em dois momentos diferenciados e de grande aprendizagem.

$\mathrm{O}$ primeiro momento transcorreu ano de 2016, em um conjunto de comunidades remanescentes de quilombos, localizadas na Ilha do Marajó no estado do Pará. Atendimentos nessas comunidades demandam um aparato logístico cuidadoso, uma vez que o nível de isolamento de cada comunidade é diferenciado, em especial, no que se refere a algumas comunidades na Ilha do Marajó, o translado é esguio e composto por algumas etapas, trajeto de barco, depois van, a travessia de um rio utilizando uma embarcação rustica e a última parte, cerca de dois quilômetros realizados a pé. Por essa razão o planejamento das ações perpassa por diversos aspectos, desde a escolha do período menos chuvoso para a realização das ações e menos dificultoso para a chegada da equipe, bem como questões religiosas e culturais que podem influir na dinâmica das atividades a serem realizadas e em seus resultados.

Para conhecer a ingestão dietética dos residentes, utilizou-se um questionário de frequência alimentar (QFA), ferramenta de natureza qualitativa que tem por objetivo avaliar o consumo habitual do indivíduo conforme grupos de alimentos e bebidas específicos de acordo com o padrão dietético da população estudada (Brito et al. 2017:457-468). Além do QFA, utili- zou-se o recordatório 24horas (R24h) com o propósito de relatar o consumo de todos os alimentos e bebidas ingeridos em um prazo de 24 horas.

Dessa forma, constatou-se que determinadas frutas e verduras são ingeridas em maior quantidade conforme a safra, já as proteínas de maior frequência de consumo é a bovina e peixe fresco. O elevado consumo de alimentos ricos em carboidratos simples dentre eles o pão e a bolacha tipo cream cracker que são majoritariamente consumidos na primeira refeição do dia, mais tarde no lanche. Além do que, evidenciaram-se hábitos alimentares característicos da vida urbana, como alimentos industrializados, ultra processados e aqueles com elevados teores de açúcar refinado (Ramos et al. 2016).

Ainda nesse mesmo nicho populacional, foram encontrados altos índices de sobrepeso e obesidade na maior parte em mulheres, em uma das comunidades relatou-se o hábito de salgar alimentos para mantê-los em conservação, habito este que além de refletir o baixo poder econômico resulta no consumo elevado de sódio $(\mathrm{Na})$, fator preponderante para a incidência da hipertensão arterial sistêmica (HAS) (Nascimento et al. 2017).

O segundo momento deu-se em etnia indígena localizada no Sul do Pará, nas proximidades do Município de Marabá, em uma ação de saúde em junho de 2018 a qual contou com uma equipe multiprofissional composta nutricionistas, enfermeiros, médicos, biomédicos, e psicólogos com seus respectivos acadêmicos de cada aérea de atuação, 
proporcionando troca de saberes das práticas aplicadas em campo.

No que concerne às atividades ao campo da nutrição, observou-se uma comunidade em constante processo de "ocidentalização", com hábitos alimentares claramente influenciados pelo "homem branco", o consumo de refrigerante e produtos industrializados com elevado teor de açúcar e sódio, algo corriqueiro de se encontrar entre os relatos das opções de alimentos dos residentes.

Importante salientar que nessa ação não foi realizado procedimentos de avaliação do padrão de consumo alimentar como o QFA e R24h por serem métodos que necessitam de um tempo maior para sua aplicação, contudo, a sensibilidade no atendimento e contemplação da população nos permitiu perceber tais padrões já enraizados em paralelo com seus hábitos alimentares específicos. Convém neste momento também deixar claro que o padrão alimentar de etnias indígenas se diferencia entre si, e não cabe aqui uma generalização de interferência alimentar uma vez (como já relatado anteriormente) que diversas são as formas de contato que os mesmos possuem com outro tipo de população e entre outras etnias.

Em panorama geral, um elevado índice de sobrepeso e obesidade, e sua maioria no sexo feminino condição similar evidenciado no sexo feminino nas comunidades quilombolas anteriormente narrados, os quais são fatores de risco para doenças crônicas não transmissíveis como diabetes e hipertensão (Claro et al. 2015:257-265).
A compreensão do consumo alimentar em distintas populações transcorre na herança cultural e valor histórico do alimento ingerido, tais temáticas são redesenhadas constantemente por processos sociais e migratórios, somando-se a essas temáticas tem-se a determinação dos hábitos alimentares consequentes dos recursos naturais e econômicos (Jaime et al. 2015:267-276).

Em ambas as visitas se constatou a vulnerabilidade extrínseca que afeta esses grupos, uma vez que ficou perceptível a influência que estes recebem, em suas vestimentas, maneira de dialogar e principalmente em seus hábitos alimentares, os quais refletiam na situação de saúde. Perceber estas características e peculiaridades possibilita originar estratégias efetivas em educação e saúde.

A problemática que nos coloca a refletir diz respeito à deficiência que a universidade possui em não preparar o aluno de forma adequada para as especificidades amazônicas, sejam elas em povos ribeirinhos, quilombolas ou indígenas, uma vez que esta habilidade é de suma importância para a atuação apropriada do nutricionista.

Nesta perspectiva, Recine et al (2012) enfatizam a indispensabilidade de disponibilização de disciplinas que englobem a temática do SUS, a fim de prover as habilidades e competências do profissional em nutrição no domínio da análise dos problemas nutricionais tendo em conta os contextos históricos, geopolítico, ambiental e econômico de indivíduos e grupos sociais.

Além da adequação das medidas pedagógicas, manter o diálogo com os po- 
vos tradicionais da terra é crucial para a compreensão das mudanças culturais, ambientais e sociais e suas consequências no perfil de saúde, munindo o profissional de nutrição de informações adequadas para a realização do seu trabalho (Garnelo \& Pontes 2012).

\section{CONSIDERAÇÕES FINAIS}

Partindo das reflexões acima elencadas, é necessário compreender que mais que o conhecimento científico, é imprescindível se ter sensibilidade quando se atua em comunidades tradicionais. $\mathrm{O}$ olhar puramente biomédico da alimentação e saúde não se encaixa em tal realidade. É vital entender a dimensão plural destas comunidades, compreender seus hábitos de consumo, considerações que estes povos têm acerca do que é saúde e ainda, perceber como suas condições ambientais os afetam e os modificam, deixando mais suscetíveis a doenças e agravos, como também a própria perda da identidade. Para além disto, é necessário compreender que o contato com a civilização ocidental teve forte impacto e disseminou muito estas sociedades - em especial as indígenas - seja por meio de doenças, epidemias, massacres, dentre outras situações, e ainda remodelou o seu próprio modo de relação com a terra e com os seus pares. Tal rearranjo é perceptível quando se estuda a conexão com os alimentos, seja na aquisição ou o consumo, que anteriormente era um resultado de seu contato com a natureza, através da plantação ou colheita dos recursos disponíveis, e agora percebe-se que tal conseguimento se dá, muitas vezes através da compra comercial, alimentação escolar, doações em caráter emergencial e mais. Ocorre aí a inserção da relação financeira para a obtenção da alimentação, e cada vez mais os povos tradicionais se distanciam de seus hábitos e costumes. A sua relação com a Terra vai perdendo força e dando lugar a relações comerciais, agora com produtos majoritariamente industrializados, ricos em açúcar e gordura saturada e pobre em nutrientes e vitaminas, ficando visível a modificação do consumo alimentar antes rico e nutritivo, para pobre e sistematicamente maléfico, uma vez que o seu organismo não está pronto para as modificações impostas. Tais rearranjos, quando se fala de povos indígenas, têm como modificação ainda o modo como estabelecem a moradia, que antes tinha característica sazonal e temporária, e passa gradativamente a ser fixa, ocasionalmente no entorno dos Centros de Proteção ou nas reservas destinadas a estes povos, fator que dificulta a subsistência a partir da Terra, uma vez que quanto maior o tempo de permanência em um único local, maior a possibilidade de saturação do solo, tornando-a improdutiva.

Percebe-se ainda na literatura que é crescente a preocupação com os povos indígenas e quilombolas, contudo o mesmo não ocorre com as demais populações tradicionais, ocasionando uma precariedade de informações e invisibilidade demográfica e epidemiológica, uma vez que os estudos dispõem de dados destes primeiros, mas pouco se sabe dos hábitos, costumes e crenças dos demais. 
Assim, passa a ser de fundamental importância o incentivo à agrobiodiversidade, seja por meio da reforma agrária, seja na garantia de políticas públicas eficazes voltadas a estas populações, para que este público alcance o acesso ao que lhe é devido e consiga igualmente preservar sua identidade étnica e cultural. É de suma relevância ainda, o incentivo ao fortalecimento de seus costumes e hábitos alimentares, com o intuito de garantir uma alimentação rica e condizente com o seu modo de viver, desencorajando a absorção dos hábitos alimentares ocidentais maléficos, podendo tais fomentos serem realizados a partir de políticas de Estado, o que possibilitaria ganhos realmente efetivos para essas pequenas nações.

\section{REFERÊNCIAS}

Amorim, F. F. Nos taparis korubo: uma perspectiva indigenista das tensões e distensões Korubo (e Matis). 2017. Disponível em $:<$ https://povosisolados. com/2017/06/18/nos-tapiris-korubo-uma-perspectiva-indigenista-das-tensoes-e-distensoes-korubo-e-matis/> Acesso em: 18 out. 2018.

Almeida, L. D. 2010. Suscetibilidade: novo sentido para a vulnerabilidade. Revista Bioética. 18(3): 537-48.

Brasil. Ministério da Saúde. 2010. Dialogando sobre o direito bumano à alimentaşão adequada no contexto do SUS. <http://ecos-redenutri.bvs.br/cursos/curso_dialogando_online/apostila.pdf> Acesso em: 30 jun. 2018.

Brito, A. P.; Araujo, M. C.; Guimarães, C. P.; \& Pereira, R. A. 2017. Validade relativa de questionário de frequência alimentar com suporte de imagens. Ciência \& Saúde Coletiva, 22: 457-468.
2006. Lei $\mathrm{n}^{\circ} 11.346$, de 15 de setembro de 2006. Cria o Sistema Nacional de Segurança Alimentar e Nutricional Sisan com vistas em assegurar o direito humano à alimentação adequada e dá outras providências. Disponível em: https://bit. ly/2pApPdG Acesso em: 10 jul 2018.

2007. Decreto $n^{\circ}$ 6.040, de 7 de fevereiro de 2007. Institui a Política Nacional de Desenvolvimento Sustentável dos Povos e Comunidades Tradicionais. Disponível em: http://www.planalto.gov.br/ ccivil_03/_ato2007-2010/2007/decreto/ d6040.htm. Acesso em: 07 jul. 2018.

.2012. Resolução n 466 de 12 de dezembro de 2012. Aprova as Diretrizes e normas regulamentadoras de pesquisas envolvendo seres humanos. Disponível em: http://bvsms.saude.gov.br/bvs/saudelegis/cns/2013/res0466_12_12_2012.html Acesso em: 10 jul. 2018.

.2016. Resolução n 510 de 07 de abril de 2016. Dispõe sobre as Normas aplicáveis a pesquisas em Ciências Humanas e Sociais. Disponível em: http://conselho. saude.gov.br/resolucoes/2016/reso510. pdf. Acesso em: 10 jul. 2018.

Claro, R. M.; Santos, M. A. S.; Oliveira, T. P.; Pereira, C. A., Szwarcwald, C. L., \& Malta, D. C. 2015. Consumo de alimentos não saudáveis relacionados a doenças crônicas não transmissíveis no Brasil: Pesquisa $\mathrm{Na}$ cional de Saúde, 2013. Epidemiologia e Servicos de Saúde. 24: 257-265.

Conti, I. L.; Souza, G. C. 2013. Povos e comunidades tradicionais: A produção de políticas públicas de segurança alimentar e nutricional. Amazônica, Revista de Antropologia. 5 (3) Especial: 780-804.

Corradi-Perini, C.; Gonçalves, E. C.; Morimoto, I. M. I.; Ribeiro, C. D. S. G.; \& Cunha, T. R. 2018. Bioética e direito humano à alimentação adequada no contexto da terapia nutricional enteral. Revista Bioética. 26(2). 
Cosac, D. C. S. 2017. Autonomia, consentimento e vulnerabilidade do participante de pesquisa clínica. Revista Bioética. 25 (1): 19-29.

Garnelo, L.; Pontes, A. L. 2012. Saúde indígena: uma introducão ao tema. Brasília, MEC-SECADI.

Guimarães, M. C. S.; Novaes, S. C. 1999. Autonomia reduzida e vulnerabilidade: Liberdade de Decisão, Diferença e Desigualdade. Revista Bioética. <http://revistabioetica.cfm.org.br/index.php/revista_ bioetica/article/view/288/427> Acesso em: 07 jul. 2018.

Jaime, P. C.; Stopa, S. R.; Oliveira, T. P.; Vieira, M. L.; Szwarcwald, C. L.; \& Malta, D. C. 2015. Prevalência e distribuição sociodemográfica de marcadores de alimentação saudável, Pesquisa Nacional de Saúde, Brasil 2013. Epidemiologia e Serviços de Saúde. 24 (2): 267-276.

Morais, T. C. A.; Monteiro, P. S. 2017. Conceitos de vulnerabilidade humana e integridade individual para a bioética. Revista de Bioética. 25(2): 311-9.

Mattos, P. F.; Neves, A. S. 2009. A importância da atuação do nutricionista na Atenção Básica à Saúde. Revista Práxis. 1(2):11-15.

Nascimento, R. F. D.; Gavron, A. B.; Bowles, S.; Chaves, E. S.; \& Bortolozo, E. A. F. Q. 2017. Determination of the sodium and potassium levels in meals served in a university restaurant in the south of Brazil. Brazilian Journal of Food Technology. 20.

Organização das Nações Unidas. 1948. Declaração universal dos direitos bumanos. Disponível em: https://bit.ly/1yAeHbb. Acesso em: 09 jul. 2018.

Organização das Nações Unidas para a Educação, a Ciência e a Cultura - Unesco. 2005. Declaração universal sobre bioética e direitos humanos. Disponível em: https://bit. ly/1TRJFa9 Acesso em: 10 jul. 2018.
Ramos, E. M. L. S; Almeida, S. S; Araújo, A. R.; Santos, R. P.; Arede, A. N. F.; Lima, E. J. S. 2016. Métodos e Ações Nutricionais em Quilombos. Praia, Edições: Uni-CV.168p.

Rebelo, F. P. 2014. Os aspectos legais da territorialização no Brasil: Algumas reflexões sobre o conceito de populações tradicionais. Diálogo. 27: 75-84.

Recine, E.; Gomes, R. C. F.; Romeiro, A. A. F.; Pinheiro, A. R. D. O.; Teixeira, B. D. A.; Sousa, J. S. D. 2012. A formação em saúde pública nos cursos de graduação de nutrição no Brasil. Revista de Nutrição. 25(1):21-33.

Rogers, W.; \& Ballantyne, A. 2008. Populações especiais: vulnerabilidade e proteção. RECIIS: Revista Eletrônica de Comunicação, Informação e Inovação em Saúde. 2(1): 30-41.

Silva, H. P. 2006. A Saúde Humana e a Amazônia no Século XXI: Reflexões Sobre os Objetivos do Milênio. Novos Cadernos do NAEA/UFPA, 9(1):77-94.

Soares, D. A.; Barreto, S. M. 2014. Sobrepeso e obesidade abdominal em adultos quilombolas, Bahia, Brasil. Cadernos de Saúde Pública: 30:341-354. 